\title{
VARIETAL IMPROVEMENT OF NORMAL SEASON IRRIGATED RICE FOR EASTERN TERAI REGION OF NEPAL
}

\author{
Hari Kumar Prasai ${ }^{1}$, Rudra Bhattarai ${ }^{1}$ and Jiban Shrestha ${ }^{2}$ \\ ${ }^{1}$ Regional Agricultural Research Station, Tarahara, Sunsari, Nepal \\ ${ }^{2}$ National Maize Research Program, Rampur, Chitwan, Nepal \\ Corresponding address: hkprasai60@gmail.com
}

\begin{abstract}
The coordinated and regional varietal trial of normal season irrigated rice was carried out at Regional Agricultural Research Station (RARS), Tarahara, Nepal in 2014 \& 2015. Out of the tested genotypes, NR 2157-166-1-3-5-1, Cieharang sub-1 and NR 2157-166-2-1-1-1 identified as promising with potential grain yield of 4313,4290 and $4239 \mathrm{~kg} / \mathrm{ha}$ respectively in over year analysis (2014-15) of coordinated varietal trial (CVT). These genotypes recorded 25 to 27 percent higher grain yield than national rice productivity and 4 to 6 percent more than check variety, Sabitri. The differences in grain yield among the tested genotypes and between the years were found significant, but interaction of genotypes to year was found non-significant. Similarly, KARAJAT-3 recorded the highest grain yield (4897 kg/ha) followed by NR 1190-24-4-2-2-2-3$2(4861 \mathrm{~kg} / \mathrm{ha})$ in over year analysis (2014-15) of regional varietal trial (RVT), similarly and identified as promising genotypes. These promising genotypes recorded 43 and 8 percent higher grain yield than national rice yield and to the check variety, Sabitri, respectively in over year analysis. The differences in grain yield among genotypes and year were found significant.
\end{abstract}

Keywords: Genotypes; significant; normal rice; promising; grain yield; kg/ha; CVT; RVT

\section{Introduction}

Rice (Oryza sativa $\mathrm{L}$ ) is the major staple cereal crops of the country. It occupies 42.72 percent area (1.49 million hectares) of the total cultivated area of the cereal crops (3.48 million hectares) and about 53 percent production (5.048 million tons) of the total production ( 9.56 million tons) of the cereal crops of the country. The average rice productivity of the nation is $3394 \mathrm{~kg} / \mathrm{ha}$ whereas it is 3586 $\mathrm{kg} / \mathrm{ha}$ for eastern Terai region (MOAD, 2014).

Rice cultivated area and production of the country is dominated by Terai region. About 68 percent rice cultivated area and 70 percent rice production is contributed by Terai region (MOAD, 2014). About 70 percent of the total rice is produced for household consumption (Ghimire et al., 2013).

Globally, rice is cultivated from latitude $35^{\circ} \mathrm{S}$ to $50^{\circ} \mathrm{N}$ and from sea level to 3000 meter as well as in both temperate and tropical climates. Rice can be grown in a variety of water regimes, that is, un submerged upland rice, moderately submerged lowland rice and submerged rice. Similarly, It can be grown in a wide range of soil types (Takahashi, 1984, Oka, 1988, OECD, 1999). In Nepal, rice is being cultivating in diverse ecosystem such as irrigated
$(51 \%)$, upland (9\%), rainfed lowland (37\%), flash flood and deep water (3\%) (Yadav et al., 2013).

Rice is one of the most important staple foods for more than half of the world's population and influences the livelihoods and economics of several billion peoples (IRRI, 2006) which provides $20 \%$ of the per capita energy and $13 \%$ of the protein consumed worldwide (Juliano, 1994).

These experiments were carried out in Regional Agricultural Research Station (RARS), Tarahara, Sunsari, Nepal in 2014 and 2015. This research station is located at $26^{\circ} 42^{\prime}$ North latitude and $87^{\circ} 16^{\prime}$ East longitude, and 136 meter above sea level. The climatic condition of the research farm is sub-tropical and soil texture is dominated by clay loam and $\mathrm{pH}$ of the soil ranges from 6.5-7.0 (RARS, 2014). Similarly, The temperature ranges from minimum to maximum of $9.3^{\circ} \mathrm{C}$ (January) and $35.5^{\circ} \mathrm{C}$ (April) in 2014 while it was $9.8^{\circ} \mathrm{C}$ (January) and $33.5^{\circ} \mathrm{C}$ (July) in 2015 respectively. Similarly, annual precipitation in was found higher $(2031.1 \mathrm{~mm})$ in 2015 than in $2014(1653.8 \mathrm{~mm})$. The occurrence of rainfall from rice transplanting to flowering was higher $(1281.8 \mathrm{~mm})$ in 2015 whereas it was $916.2 \mathrm{~mm}$ in 2014 . 
Rice variety development activities had initiated to develop high yielding rice varieties for irrigated ecosystem in 1980s (Mallik, 1981). Total 50 varieties had already been released from 1967 to 2011 (MOAD, 2014). Effective agriculture research and extension is one of the key components in increasing agriculture productivity (ADS, 2014). Agricultural genetics is one of the components of increasing food production to meet the demand of ever increasing population (Editorial Nature Genetics, 2009). Positive changes in today agriculture became possible only through the development of technologies. In addition, these experiments were designed to identify high yielding genotypes as irrigated rice for eastern Terai of Nepal.

\section{Materials and Methods}

The CVT of normal season rice was carried out in 2014 and 2015 at irrigated condition of RARS, Tarahara, Sunsari Nepal. Randomized Complete Block Design (RCBD) was applied as designing of the experiments with three replications in each year. The plot size of $10 \mathrm{~m}^{2}$ was maintained and transplanted in spacing of $20 \times 20 \mathrm{~cm}^{2}$ in both years. CVT experiment of 2014 was seeded in 26 June, 2014 and transplanted in 25 July, 2014. Total 30 genotypes namely, IR 70215-39-CPA, IR 79525-20-2-2-2, HUANZHUAZHA, NR 2157-63-2-2-1-1, IR 87377-B-B93-3, IR 76939-98-1-1-1, NR 2158-13-1-12-4, CT 191517-5-1-25R-1P, Sabitri, NR 2167-46-1-1-1-1, NR 2157-1662-1-1-1, NR 2157-32-2-2-2-1, NR 2157-32-2-2-2-1, NR 2157-166-1-3-5-1, IR 83372-B-B-33-2, NR 2157-198-1-32-1, MTU 115, IR 79584-38-2-1-4, Cieharang Sub-1, NR 2157-144-1-3-1-1-1, NR 2158-13-1-2-4-1, NR 2157-54-11-1-1, IR 70210-39-CPA-7-1, 8NR2167-41-11-3-1, HWA 564, IR 11F 267, IR 73718-26-1-2-5, CT 1848-6-9-3-3-2, IR 80285-34-3-3-2, YN 2883-12-2-1 and IR 78875-207-BB-B were included in the experiment of 2014.

Similarly, the CVT of 2015 was seeded in 28 June, 2015 and transplanted in 25 July, 2015. Total 24 genotypes namely, IR05N-304, NR 2158-13-1-12-4, IR 73718-26-12-5, IR 94391-131-358-19-B-1-1-1, IR 80285-34-3-3-2, NR 2157-66-2-3-1-1-1, IR 96321-1099-227-B-3-1-3, CIEHARANG SUB-1, IR 78875-207-B-B-B, IR 79584-382-1-4, IR 87377-B-B-93-3, IR10A277, NR 2157-32-2-1-11-1, CT 19151-7-5-125R-1P, ZR 2167-48-5-1-2-1-1, IR 79525-20-2-2-2, NR 2157-166-1-3-5-1, IR 96322-34-202B-2-1-2, NR 2158-13-1-2-4-1, NR 2157-166-2-1-1-1, NR 2157-144-1-3-1-1, SABITRI, IR 96321-558-64-B-4-1-1 and NR 2167-41-1-1-3-1 were included in the CVT of 2015. The fertilizers were applied at the rate of 100:40:30 NPK $\mathrm{kg} / \mathrm{ha}$. Complete dose of phosphorus and potassium, half dose of nitrogenous fertilizers were applied as basal dose and remaining half dose of nitrogenous fertilizer was applied as top dress in two split doses, that is, 25 and 45 days after transplanting in both years. Days to heading and maturity, plant height, panicle length, tillers $/ \mathrm{m}^{2}$, number of filled and unfilled grains in panicle, and grain yield were studies in both years' experiment. MSTAT-C and Excel software were used in analyzing the recorded data.

Regional varietal trial (RVT) of normal season irrigated rice was also conducted in 2014 and 2015 at RARS, Tarahara. The RVT was composed with the promising genotypes selected from CVT. RCBD was followed in layout of the experiment and the experiments were replicated three times in both years. The plot size, fertilizers dose and applications were same as above mentioned in CVT. The seeding of RVT was done in 26 June, 2014 and it was transplanted in July 25, 2014 in 2014 whereas seeding and transplanting was done in 28 June, 2015 and 20 July, 2015 respectively in 2015. Total 22 entries namely, ZX 115, IR 78555-68-3-3-36, SACG-7, IR 77721-93-2-2-1-2, IR 75299-94-1-2-2, IR 73007-131-1-2-2, IR 78875-207-B-B-B, IR 73930-31-3-22, IR 70215-70-CPA-4-1-3, JH15-1-1-1, OM 576, IR08N136, Karajat-3, IR 87377-B-B-93-3, IR 79246-1052-2-4, BRRI Dhan-38, Samba Masuli Sub-1, Swarna Sub1, Sabitri, Kanchhi Masuli, NR 1190-24-4-2-2-2-3-2 and Cieharang Sub-1 were included in the experiment of 2014. Similarly, 22 genotypes were also included in RVT of 2015, they were: IR 75299-94-1-2-2, IR 73007-131-1-2-2, IR 78875-207-B-B-B, IR 70215-70-CPA-4-1-1-3, IR08N 136, KARAJAT-3, IR 87377-B-B-93-3, IR 79246-105-2-2-4, NR 2158-13-1-2-4-1, MTU 115, NR 2157-32-2-2-2-1, NR 2167-41-11-3-1, NR 2158-13-1-12-4, NR 2167-46-1-1-1-1, NR 2157-166-2-1-1-1, BRRI DHAN-38, Samba Masuli Sub-1, Swarna Sub-1, Sabitri, Kanchhi Masuli, NR190-244-2-2-2-3-2 and Cieharang Sub-1. The same characters as mentioned above in CVT were studied in RVT also and same software was used to analyze the data.

\section{Result and Discussion}

\section{Coordinated Varietal Trial}

Out of the tested CVT genotypes in 2014, IR 70215-39CPA genotype was found early in flowering (97.66 days) and similar to maturity (126.67 days), but it produced lowest yield (2583 kg/ha). NR 2157-54-1-1-1genotype took long day to flower (123 days) and mature (151.33 days). The lowest plant height $(90.33 \mathrm{~cm})$ was observed for NR 2157-198-1-3-2-1 and highest plant height (121.33 cm) was observed for IR 70210-39-CPA-7-1. Similarly, NR 215813-1-2-4-1 produced the highest grain yield $(4125 \mathrm{~kg} / \mathrm{ha})$ followed by MTU 115 (4083 kg/ha), IR 76939-98-1-1-1 and NR 2157-32-2-2-2-1 (4000 kg/ha). These genotypes recorded 4 and 17 percent higher grain yield than check variety Sabitri $(3833 \mathrm{~kg} / \mathrm{ha})$ and national productivity of rice respectively. Likewise, NR 2157-144-1-3-1-1, IR 8028534-3-3-2, 8NR2167-41-11-3-1 and NR 2157-54-1-1-1-1 genotypes produced 2 and $15 \%$ higher grain yield than check variety and national productivity of rice and these 6 high yielding genotypes identified as superior from CVT, 2014. All of the characters included in these experiment were found significantly different among the tested genotypes (Table 1). 
Table1: Grain yield and other ancillary characters of rice in coordinated varietal trial of 2014

\begin{tabular}{|c|c|c|c|c|c|c|c|}
\hline S.N. & Genotypes & $\begin{array}{l}\text { Flowering } \\
\text { days }\end{array}$ & $\begin{array}{l}\text { Maturity } \\
\text { days }\end{array}$ & $\begin{array}{l}\text { Plant height } \\
(\mathrm{cm})\end{array}$ & Tillers/m $\mathbf{m}^{2}$ & $\begin{array}{l}\text { Panicle } \\
\text { length }(\mathrm{cm}) \\
\end{array}$ & $\begin{array}{l}\text { Grain yield } \\
\text { (kg/ha) }\end{array}$ \\
\hline 1 & IR 70215-39-CPA & 97.66 & 126.67 & 112.67 & 186.33 & 23.00 & 2583.00 \\
\hline 2 & IR 73718-26-1-2-5 & 106.66 & 132.33 & 110.33 & 212.66 & 24.00 & 3666.00 \\
\hline 3 & HUANZHUAZHA & 101.66 & 130.67 & 99.33 & 208.00 & 23.67 & 3458.00 \\
\hline 4 & NR 2157-166-1-3-5-1 & 119.67 & 147.67 & 102.00 & 211.67 & 23.33 & 3208.00 \\
\hline 5 & NR 2157-63-2-2-1-1 & 109.33 & 138.33 & 98.67 & 230.00 & 22.33 & 3125.00 \\
\hline 6 & NR 2157-166-2-1-1-1 & 119.00 & 147.00 & 101.67 & 235.67 & 23.67 & 3708.00 \\
\hline 7 & NR 2158-13-1-2-4-1 & 113.66 & 141.33 & 97.67 & 235.67 & 23.00 & 4125.00 \\
\hline 8 & 8NR2167-41-11-3-1 & 112,67 & 141.67 & 118.00 & 229.33 & 24.00 & 3916.00 \\
\hline$y$ & $\begin{array}{l}\text { CT 19151-7-5-1-25R- } \\
\text { 1P }\end{array}$ & 108.00 & 136.66 & 106.33 & 204.00 & 24.67 & 3125.00 \\
\hline 10 & IR 76939-98-1-1-1 & 116.67 & 144.67 & 112.00 & 243.33 & 25.67 & 4000.00 \\
\hline 11 & YN 2883-12-2-1 & 107.67 & 136.67 & 102.67 & 197.00 & 24.67 & 3208.00 \\
\hline 12 & IR 80285-34-3-3-2 & 104.33 & 133.33 & 104.67 & 208.67 & 26.67 & 3958.00 \\
\hline 13 & NR 2157-198-1-3-2-1 & 111.67 & 140.33 & 90.33 & 247.33 & 21.67 & 3666.00 \\
\hline 14 & NR 2167-46-1-1-1-1 & 112.67 & 141.66 & 125.33 & 241.00 & 25.00 & 3666.00 \\
\hline 15 & HWA-564 & 106.33 & 135.33 & 117.00 & 150.33 & 26.33 & 3458.00 \\
\hline 16 & NR 2157-54-1-1-1-1 & 123.00 & 151.33 & 105.33 & 204.33 & 23.33 & 3916.00 \\
\hline 17 & NR 2157-32-2-2-2-1 & 120.67 & 148.67 & 134.33 & 240.33 & 24.00 & 4000.00 \\
\hline 18 & NR 2157-144-1-3-1-1 & 112.67 & 141.00 & 99.00 & 223.33 & 23.67 & 3958.00 \\
\hline 19 & IR 78875-207-B-B-B & 101.67 & 130.67 & 102.33 & 204.67 & 23.33 & 3291.00 \\
\hline 20 & IR 83372-B-B-33-2 & 99.67 & 128.67 & 116.67 & 199.90 & 23.67 & 3291.00 \\
\hline 21 & NR 2158-13-1-12-4 & 113.67 & 141.67 & 104.67 & 235.00 & 23.67 & 3916.00 \\
\hline 22 & IR 79525-20-2-2-2 & 106.32 & 135.33 & 108.00 & 236.00 & 24.67 & 3291.00 \\
\hline 23 & Cieharang Sub-1 & 104.67 & 133.67 & 99.00 & 226.33 & 23.33 & 3833.00 \\
\hline 24 & CT 1848-6-9-3-3-2 & 105.00 & 134.00 & 103.67 & 210.33 & 24.33 & 3625.00 \\
\hline 25 & IR 87377-B-B-93-3 & 102.33 & 131.33 & 115.33 & 202.33 & 23.00 & 3083.00 \\
\hline 26 & $\begin{array}{l}\text { IR 70210-39-CPA-7- } \\
1\end{array}$ & 100.00 & 129.00 & 121.33 & 217.67 & 25.00 & 3166.00 \\
\hline 27 & IR 79584-38-2-1-4 & 101.00 & 130.00 & 107.00 & 224.67 & 25.33 & 3416.00 \\
\hline 28 & MTU 115 & 113.00 & 142.00 & 102.67 & 179.33 & 25.00 & 4083.00 \\
\hline 29 & IR11F 267 & 106.00 & 135.00 & 99.33 & 231.33 & 24.00 & 3541.00 \\
\hline \multirow[t]{5}{*}{30} & Sabitri & 117.00 & 145.00 & 108.67 & 250.33 & 25.00 & 3833.00 \\
\hline & Grand Mean & 109.14 & 137.72 & 107.53 & 217.53 & 24.10 & 3570.00 \\
\hline & F test & $* *$ & $* *$ & $* *$ & $*$ & $* *$ & $* *$ \\
\hline & $\mathrm{CV} \%$ & 1.64 & 1.46 & 4.75 & 13.58 & 4.57 & 11.79 \\
\hline & LSD (0.05) & 3.89 & 4.38 & 11.12 & 63.88 & 2.397 & 915.30 \\
\hline
\end{tabular}

Note: $* *=p<0.01, *=p<0.05$

Among the tested genotypes in CVT of 2015, IR 79584-382-1-4 and IR87377-B-B-93-2 were found early in maturity (125.67 days) with $4.0-4.2$ t/ha grain yield whereas IR
96321-1099-227-B-3-1-3 was observed as most late in maturity (149 days). Genotype IR 94391-131-358-19-B-11-1 produced the highest grain yield $(5504 \mathrm{~kg} / \mathrm{ha})$ followed 
by IR 96322-34-202-B-2-1-2 (5436 kg/ha), IR 96321-1099227-B-3-1-3 (5047 kg/ha), CT 19151-7-5-1-25R-1P (5024 $\mathrm{kg} / \mathrm{ha}$ ) and NR 2157-66-2-3-1-1-1 (5018 kg/ha). The maturity of top yielder genotypes IR 94391-131-358-19-B1-1-1 and IR 96322-34-202-B-2-1-2 were found 133 and
139 days respectively. Total 13 genotypes recorded 34 to 60 percent higher grain yield than national productivity of rice. All tested characters except panicle length was found significantly different due to genotypes (Table 2)

Table 2: Grain yield and other ancillary characters of rice in coordinated varietal trial of 2015

\begin{tabular}{|c|c|c|c|c|c|c|c|}
\hline S.N. & Genotypes & $\begin{array}{l}\text { Flowering } \\
\text { days }\end{array}$ & $\begin{array}{l}\text { Maturity } \\
\text { days }\end{array}$ & $\begin{array}{l}\text { Plant height } \\
\text { (cm) }\end{array}$ & Tillers $/ \mathbf{m}^{2}$ & $\begin{array}{l}\text { Panicle length } \\
\quad(\mathrm{cm})\end{array}$ & $\begin{array}{l}\text { Grain yield } \\
\text { (kg/ha) }\end{array}$ \\
\hline 1 & IR 79525-20-2-2-2 & 101.33 & 129.00 & 102.87 & 206.67 & 25.20 & 4282.00 \\
\hline 2 & IR 87379-B-B-93-2 & 97.67 & 125.67 & 108.93 & 199.67 & 25.33 & 4058.00 \\
\hline 3 & NR 2158-13-1-12-4 & 113.67 & 140.00 & 94.73 & 230.67 & 22.87 & 4351.00 \\
\hline 4 & $\begin{array}{l}\text { CT 19151-7-5-1-25R- } \\
1 \mathrm{P}\end{array}$ & 108.33 & 136.33 & 114.40 & 241.67 & 24.00 & 5024.00 \\
\hline 5 & NR 2157-166-2-1-1-1 & 109.33 & 140.33 & 98.67 & 230.00 & 24.13 & 4770.00 \\
\hline 6 & NR 2157-166-1-3-5-1 & 112.67 & 140.00 & 98.67 & 222.33 & 23.40 & 4183.00 \\
\hline 7 & IR 79584-38-2-1-4 & 97.67 & 125.67 & 105.67 & 183.67 & 24.20 & 4286.00 \\
\hline 8 & NR 2157-144-1-3-1-1 & 105.66 & 133.67 & 98.87 & 202.00 & 23.13 & 4150.00 \\
\hline 9 & NR 2158-13-1-2-4-1 & 109.33 & 137.00 & 92.53 & 234.00 & 23.27 & 4899.00 \\
\hline 10 & NR 2167-41-1-1-3-1 & 108.33 & 136.33 & 112.26 & 230.67 & 22.93 & 4918.00 \\
\hline 11 & IR 73718-26-1-2-5 & 103.33 & 131.00 & 103.06 & 237.00 & 24.40 & 3603.00 \\
\hline 12 & IR 80285-34-3-3-2 & 102.33 & 130.00 & 95.73 & 213.33 & 24.87 & 4061.00 \\
\hline 13 & IR 78875-207-B-B-B & 102.00 & 129.67 & 97.73 & 206.67 & 24.00 & 2918.00 \\
\hline 14 & Cieharang Sub-1 & 98.33 & 126.33 & 99.20 & 191.00 & 23.87 & 4747.00 \\
\hline 15 & NR 2157-32-2-1-1-1-1 & 110.33 & 138.00 & 114.86 & 260.00 & 22.40 & 4724.00 \\
\hline 16 & IR05N 304 & 104.66 & 132.67 & 97.13 & 228.67 & 23.07 & 4451.00 \\
\hline 17 & NR 2167-48-5-1-2-1-1 & 119.67 & 146.67 & 125.46 & 208.67 & 23.13 & 4890.00 \\
\hline 18 & NR 2157-66-2-3-1-1 & 119.67 & 146.33 & 111.80 & 255.33 & 24.40 & 5018.00 \\
\hline 19 & $\begin{array}{l}\text { IR 96321-558-64-B-4- } \\
1-1\end{array}$ & 109.33 & 137.33 & 102.27 & 200.00 & 24.33 & 4814.00 \\
\hline 20 & $\begin{array}{l}\text { IR 94391-131-358-19- } \\
\text { B-1-1-1 }\end{array}$ & 105.00 & 133.00 & 100.33 & 234.33 & 24.60 & 5504.00 \\
\hline 21 & $\begin{array}{l}\text { IR 96322-34-202-B-2- } \\
1-2\end{array}$ & 112.00 & 139.00 & 94.60 & 202.00 & 25.40 & 5436.00 \\
\hline 22 & $\begin{array}{l}\text { IR 96321-1099-227- } \\
\text { B-3-1-3 }\end{array}$ & 112.67 & 149.00 & 101.13 & 215.00 & 25.07 & 5047.00 \\
\hline 23 & IR10A 277 & 98.33 & 126.33 & 106.46 & 192.00 & 24.27 & 4548.00 \\
\hline \multirow[t]{5}{*}{24} & Sabitri & 110.67 & 138.67 & 101.87 & 205.33 & 25.27 & 4335.00 \\
\hline & Grand Mean & 107.18 & 135.33 & 103.30 & 217.86 & 24.06 & 4553.00 \\
\hline & F test & $* *$ & $* *$ & $* *$ & $* *$ & NS & $* *$ \\
\hline & $\mathrm{CV} \%$ & 4.43 & 2.24 & 4.35 & 10.07 & 7.24 & 13.25 \\
\hline & LSD (0.05) & 10.41 & 6.639 & 9.856 & 48.14 & & 1320.00 \\
\hline
\end{tabular}

Note: $* *=p<0.01$ 
Over year analysis of the tested characters included in CVT of 2014 and 2015 was also carried out. IR 78875-207-B-B$\mathrm{B}$ was found earliest maturing (127.67 days) genotype but the grain yield was low $(2883 \mathrm{~kg} / \mathrm{ha})$ than the national productivity (3394 kg/ha). IR 87377-B-B-93-3 and IR 79584-38-2-1-4 genotypes were found second early maturing genotypes (128-129 days) and produced 5-9 percent more grain yield ( 3570 and $3701 \mathrm{~kg} / \mathrm{ha}$ respectively) than national yield of rice. NR 2158-13-1-2-4-1 genotype produced the highest grain yield $(4313 \mathrm{~kg} / \mathrm{ha})$ followed by
Cieharang Sub-1 (4290 kg/ha) and NR 2157-166-2-1-1-1 (4239 kg/ha). These high yielding genotypes recorded about 4-6 and 25-27 percent more grain yield than check variety Sabitri and national yield of rice respectively. Maturity period of these genotypes was found 139, 130 and 143 days respectively. The difference in days to flowering and maturity, plant height and grain yield was found significant among the genotypes and year, but interaction of genotypes to year was observed non-significant (Table 3).

Table 3: Combined analysis of grain yield and other ancillary characters of rice in CVT of 2014-15

\begin{tabular}{|c|c|c|c|c|c|c|c|c|}
\hline S.N. & Genotypes & $\begin{array}{l}\text { Flowering } \\
\text { days }\end{array}$ & $\begin{array}{l}\text { Maturity } \\
\text { days }\end{array}$ & $\begin{array}{l}\text { Plant } \\
\text { height }(\mathrm{cm})\end{array}$ & Tillers $/ \mathbf{m}^{2}$ & $\begin{array}{l}\text { Panicle } \\
\text { length }(\mathrm{cm})\end{array}$ & $\begin{array}{l}\text { Grain filling } \\
\text { percent }\end{array}$ & $\begin{array}{l}\text { Grain yield } \\
\text { (kg/ha) }\end{array}$ \\
\hline 1 & IR 73718-26-1-2-5 & 105.00 & 131.67 & 107.53 & 218.83 & 24.53 & 87.26 & 3864.00 \\
\hline 2 & NR 2157-166-1-3-5-1 & 116.16 & 143.83 & 100.33 & 217.00 & 23.36 & 86.81 & 3695.00 \\
\hline 3 & NR 2157-166-2-1-1-1 & 114.16 & 143.67 & 100.17 & 232.83 & 23.90 & 87.77 & 4239.00 \\
\hline 4 & NR 2158-13-1-2-4-1 & 111.50 & 139.16 & 95.20 & 234.83 & 23.13 & 86.36 & 4313.00 \\
\hline 5 & IR $80285-34-3-3-2$ & 103.33 & 131.66 & 100.20 & 211.00 & 25.76 & 82.43 & 4009.00 \\
\hline 6 & NR 2157-144-1-3-1-1 & 109.17 & 137.33 & 98.93 & 214.50 & 23.40 & 86.94 & 4054.00 \\
\hline 7 & IR 78875-207-B-B-B & 99.16 & 127.67 & 102.20 & 185.50 & 23.73 & 87.68 & 2883.00 \\
\hline 8 & NR 2158-13-1-12-4 & 113.67 & 140.83 & 99.70 & 232.83 & 23.27 & 85.03 & 4134.00 \\
\hline 9 & IR 79525-20-2-2-2 & 103.83 & 132.17 & 105.43 & 221.33 & 24.93 & 84.88 & 3787.00 \\
\hline 10 & Cieharang & 101.50 & 130.00 & 99.10 & 208.66 & 23.60 & 90.37 & 4290.00 \\
\hline 11 & IR 87377-B-B-93-3 & 100.00 & 128.50 & 112.13 & 200.16 & 23.33 & 86.61 & 3570.00 \\
\hline 12 & IR 79584-38-2-1-4 & 100.67 & 129.17 & 105.10 & 211.00 & 25.23 & 81.55 & 3701.00 \\
\hline 13 & Sabitri & 113.83 & 141.83 & 105.27 & 226.33 & 25.13 & 88.44 & 4084.00 \\
\hline & Grand Mean & 107.07 & 135.19 & 102.40 & 216.52 & 24.10 & 86.32 & 3894.00 \\
\hline & F test Genotypes & $* *$ & $* *$ & $* *$ & NS & $* *$ & NS & $* *$ \\
\hline & Year & $* *$ & $* *$ & $* *$ & NS & NS & NS & $* *$ \\
\hline & Genotypes x Year & NS & NS & NS & NS & NS & NS & NS \\
\hline & $\mathrm{CV} \%$ & 2.54 & 1.94 & 4.04 & 5.80 & 5.61 & 5.80 & 14.02 \\
\hline & $\operatorname{LSD}(0.05)$ & 4.212 & 4.059 & 6.393 & & 2.09 & & 844.4 \\
\hline
\end{tabular}

\section{Regional Varietal Trial}

Out of the tested genotypes included in RVT of normal irrigated rice during 2014, BRRI Dhan - 38 was found early in maturity (121 days) and shortest panicle length (21.33 $\mathrm{cm})$, it produced yield by 27 percent $(2483 \mathrm{~kg} / \mathrm{ha})$ than national average grain yield of rice. The highest $(143 \mathrm{~cm})$ and lowest $(91 \mathrm{~cm})$ plant height was found in NR-1190-244-2-2-2-3-2 and Samba Masuli Sub-1 respectively. IR
7025-70-CPA-4-1-1-3 produced the highest grain yield (4416 kg/ha) followed by NR-1190-24-4-2-2-2-3-2 (4250 $\mathrm{kg} / \mathrm{ha}$ ). These genotypes recorded 25-30 and 3-30 percent higher grain yield than national yield of rice and check variety Sabitri, respectively and identified as superior genotypes. All the tested traits except number of tillers $/ \mathrm{m}^{2}$ were found different significantly due to genotypes (Table 4) 
Table 4: Grain yield and other ancillary characters of rice in regional varietal trial of 2014

\begin{tabular}{|c|c|c|c|c|c|c|c|}
\hline S.N. & Genotypes & $\begin{array}{l}\text { Flowering } \\
\text { days }\end{array}$ & $\begin{array}{l}\text { Maturity } \\
\text { days }\end{array}$ & $\begin{array}{l}\text { Plant height } \\
(\mathrm{cm})\end{array}$ & Tillers $/ \mathrm{m}^{2}$ & $\begin{array}{l}\text { Panicle length } \\
(\mathrm{cm})\end{array}$ & $\begin{array}{l}\text { Grain yield } \\
\text { (kg/ha) }\end{array}$ \\
\hline 1 & ZX 115 & 103.33 & 132.33 & 106.00 & 226.00 & 25.00 & 4041.00 \\
\hline 2 & IR 78555-56-3-3-3 & 104.00 & 133.00 & 108.67 & 198.00 & 24.00 & 3037.00 \\
\hline 3 & SACG-7 & 101.33 & 130.33 & 102.33 & 204.33 & 25.67 & 3125.00 \\
\hline 4 & IR 77721-93-2-2-1-2 & 106.67 & 135.67 & 104.67 & 157.33 & 23.67 & 3625.00 \\
\hline 5 & IR 75299-94-1-2-2 & 104.33 & 133.33 & 107.67 & 196.00 & 25.00 & 3416.00 \\
\hline 6 & IR 73007-131-1-2-2 & 105.67 & 134.67 & 106.67 & 188.00 & 26.33 & 3666.00 \\
\hline 7 & IR 78875-207-B-B-B & 101.00 & 130.00 & 106.67 & 248.33 & 25.33 & 3500.00 \\
\hline 8 & IR 73930-31-3-2-1 & 102.33 & 131.33 & 110.00 & 212.33 & 25.67 & 3250.00 \\
\hline \multirow[t]{2}{*}{9} & IR 70215-70-CPA-4- & 111.33 & 140.33 & 104.33 & 200.66 & 26.00 & 4416.00 \\
\hline & $1-1-3$ & & & & & & \\
\hline 10 & JH 15-1-1-1 & 104.67 & 133.67 & 105.33 & 180.33 & 24.00 & 4166.00 \\
\hline 11 & OM 576 & 101.33 & 130.33 & 95.33 & 212.00 & 23.33 & 3708.00 \\
\hline 12 & IR08N-136 & 107.33 & 136.00 & 108.00 & 183.33 & 26.33 & 4083.00 \\
\hline 13 & KARJAT-3 & 106.67 & 135.67 & 101.33 & 183.33 & 25.00 & 4125.00 \\
\hline 14 & IR 87377-B-B-93-3 & 101.33 & 130.00 & 108.33 & 250.33 & 23.66 & 3500.00 \\
\hline 15 & IR 79246-105-2-2-4 & 110.33 & 139.33 & 108.00 & 135.33 & 26.66 & 3500.00 \\
\hline 16 & BRRI Dhan-38 & 92.00 & 121.00 & 102.33 & 214.66 & 21.33 & 2483.00 \\
\hline 17 & Samba Masuli Sub-1 & 114.67 & 142.67 & 91.00 & 212.66 & 24.00 & 3083.00 \\
\hline 18 & Swarna Sub-1 & 119.00 & 147.00 & 92.67 & 183.33 & 22.33 & 3666.00 \\
\hline 19 & Sabitri & 111.67 & 138.67 & 104.67 & 208.66 & 26.33 & 4125.00 \\
\hline 20 & Kanchhi Masuli & 110.33 & 139.33 & 110.33 & 236.00 & 23.33 & 4210.00 \\
\hline \multirow[t]{2}{*}{21} & IR 1190-24-4-2-2-2- & 115.33 & 143.00 & 143.00 & 172.66 & 24.33 & 4250.00 \\
\hline & $3-2$ & & & & & & \\
\hline 22 & Cieharang Sub-1 & 102.33 & 131.33 & 99.67 & 211.33 & 22.00 & 3818.00 \\
\hline & Grand Mean & 106.22 & 134.95 & 115.73 & 205.22 & 24.51 & 3672.00 \\
\hline & F test & $* *$ & $*$ & $* *$ & NS & $* *$ & $* *$ \\
\hline & $\mathrm{CV} \%$ & 1.77 & 1.35 & 5.06 & 19.21 & 6.05 & 9.00 \\
\hline & $\operatorname{LSD}(0.05)$ & 4.139 & 4.012 & 11.78 & & 3.266 & 728.10 \\
\hline
\end{tabular}


Similarly 22 genotypes were tested in RVT of 2015, out of which, BARRI Dhan - 38 identified as early maturing genotype (119.67 day) and second shortest panicle length $(22.27 \mathrm{~cm})$, but it's grain production was found about 4 percent low $(3272 \mathrm{~kg} / \mathrm{ha})$ compared to national average yield. NR1190-24-4-2-2-2-3-2 was found tallest (136.20 $\mathrm{cm})$ genotype whereas Cieharang Sub-1 was found dwarf (90.13 cm). Regarding grain yield, NR 2167-41-11-3 produced the highest grain yield $(4591 \mathrm{~kg} / \mathrm{ha})$ followed by KARAJAT-3 (4536 kg/ha). The maturity period of these high yielding genotypes were found 138.67 and 131.33 days respectively. These superior genotypes recorded 35 and 17 percent higher grain yield than yield of national average and check Sabitri, respectively. All the tested characters except tillers number $/ \mathrm{m}^{2}$ was found significantly different among the genotypes (Table 5).

Total thirteen genotypes were included in over year (201415) analysis of RVT. BRRI Dhan-38 was found earliest
(120.33 days) in maturity but grain yield was observed 3 percent low (3286 kg/ha) compared with national average yield. Similarly, Swarna Sub-1 was found late in maturity (147.33 days) but it yielded 28 percent more than national average and 3 percent low than check variety Sabitri. KARAJAT-3 produced the highest grain yield (4897 kg/ha) followed by NR 1190-24-4-2-2-2-3-2 (4861 kg/ha). These high yielding genotypes recorded 43 and 8 percent more grain yield than the yield of national average and check variety, Sabitri, respectively. Another check variety Kanchhi Masuli recorded $4705 \mathrm{~kg} / \mathrm{ha}$ grain yield. The grain yield of Kanchhi Masuli was observed 3 percent low than the grain yield of two high yielding genotypes. The grain filling of high yielding genotypes were found 82 and 89 percent, whereas panicle length were observed 25.30 and $25.03 \mathrm{~cm}$, respectively. The differences in maturity days, plant height, panicle length and grain yield were found significant among the genotypes and between the tested years (Table 6).

Table 5: Grain yield and other ancillary characters of rice in regional varietal trial of 2015

\begin{tabular}{|c|c|c|c|c|c|c|c|}
\hline S.N. & Genotypes & $\begin{array}{l}\text { Flowering } \\
\text { days }\end{array}$ & $\begin{array}{l}\text { Maturity } \\
\text { days }\end{array}$ & $\begin{array}{l}\text { Plant height } \\
\text { (cm) }\end{array}$ & Tillers/m² & $\begin{array}{l}\text { Panicle length } \\
(\mathrm{cm})\end{array}$ & $\begin{array}{l}\text { Grain yield } \\
\text { (kg/ha) }\end{array}$ \\
\hline 1 & IR 75299-94-1-2-2 & 102.00 & 130.00 & 106.53 & 187.33 & 25.67 & 4034.00 \\
\hline 2 & IR 73007-131-1-2-2 & 103.00 & 131.00 & 103.60 & 196.00 & 25.80 & 3517.00 \\
\hline 3 & IR 78875-207-B-B-B & 100.33 & 128.00 & 95.53 & 169.33 & 23.60 & 3717.00 \\
\hline 4 & $\begin{array}{l}\text { IR 70215-70-CPA-4- } \\
1-1-3\end{array}$ & 108.33 & 136.00 & 107.93 & 169.33 & 28.60 & 3974.00 \\
\hline 5 & IR08N136 & 104.67 & 132.67 & 100.20 & 180.33 & 25.27 & 4101.00 \\
\hline 6 & KARJAT-3 & 103.33 & 131.33 & 97.33 & 224.33 & 24.27 & 4536.00 \\
\hline 7 & IR 87377-B-B-93-3 & 102.00 & 130.00 & 105.93 & 181.00 & 23.66 & 4182.00 \\
\hline 8 & IR 79246-105-2-2-4 & 106.67 & 134.00 & 106.86 & 190.33 & 26.20 & 3374.00 \\
\hline 9 & NR 2158-13-1-2-4-1 & 109.67 & 136.67 & 91.27 & 195.67 & 22.80 & 3598.00 \\
\hline 10 & MTU-115 & 116.00 & 143.33 & 97.27 & 177.00 & 25.00 & 4232.00 \\
\hline 11 & NR 2157-32-2-2-2-1 & 115.67 & 143.00 & 125.00 & 197.33 & 23.27 & 3833.00 \\
\hline 12 & NR 2167-41-11-3-1 & 111.33 & 138.67 & 113.20 & 209.67 & 24.07 & 4591.00 \\
\hline 13 & NR 2158-13-1-12-4 & 112.67 & 140.00 & 97.40 & 181.00 & 24.07 & 4054.00 \\
\hline 14 & NR 2167-46-1-1-1-1 & 110.00 & 137.67 & 116.33 & 221.67 & 23.80 & 4284.00 \\
\hline 15 & NR 2157-166-2-1-1-1 & 110.67 & 138.33 & 101.07 & 209.67 & 22.73 & 4235.00 \\
\hline 16 & BRRI Dhan & 91.67 & 119.67 & 96.73 & 194.00 & 22.27 & 3272.00 \\
\hline 17 & Samba Masuli Sub1 & 114.67 & 141.67 & 92.80 & 212.67 & 23.53 & 3873.00 \\
\hline 18 & Swarna Sub1 & 121.00 & 147.67 & 94.33 & 219.00 & 22.27 & 4052.00 \\
\hline 19 & Sabitri & 113.33 & 141.00 & 102.33 & 213.33 & 24.93 & 3928.00 \\
\hline 20 & Kanchhi Masuli & 109.00 & 136.00 & 115.93 & 220.00 & 22.40 & 4495.00 \\
\hline 21 & $\begin{array}{l}\text { NR 1190-24-4-2-2-2- } \\
3-2\end{array}$ & 118.67 & 146.33 & 136.20 & 176.33 & 25.73 & 4378.00 \\
\hline \multirow[t]{5}{*}{22} & Cieharang Sub-1 & 103.66 & 130.67 & 90.13 & 182.67 & 22.07 & 3359.00 \\
\hline & Grand Mean & 108.50 & 136.07 & 104.28 & 195.81 & 24.18 & 3983.00 \\
\hline & F test & $* *$ & $* *$ & $* *$ & NS & $* *$ & $* *$ \\
\hline & $\mathrm{CV} \%$ & 3.06 & 2.33 & 3.59 & 14.44 & 6.11 & 9.09 \\
\hline & $\operatorname{LSD}(0.05)$ & 7.39 & 6.99 & 8.24 & & 3.256 & 797.9 \\
\hline
\end{tabular}

Note: $* *=p<0.01, *=p<0.05$ 
Table 6: Combine analysis of grain yield and other ancillary characters of rice in $2014-15$

\begin{tabular}{|c|c|c|c|c|c|c|c|c|}
\hline S.N. & Genotypes & $\begin{array}{l}\text { Flowering } \\
\text { days }\end{array}$ & $\begin{array}{l}\text { Maturity } \\
\text { days }\end{array}$ & $\begin{array}{l}\text { Plant } \\
\text { height } \\
\text { (cm) }\end{array}$ & Tillers $/ \mathrm{m}^{2}$ & $\begin{array}{l}\text { Panicle } \\
\text { length }(\mathrm{cm})\end{array}$ & $\begin{array}{l}\text { Grain } \\
\text { filling } \\
(\%)\end{array}$ & $\begin{array}{l}\text { Grain yield } \\
\text { (kg/ha) }\end{array}$ \\
\hline 1 & $\begin{array}{l}\text { IR 73007-131-1- } \\
2-2\end{array}$ & 104.33 & 132.83 & 105.03 & 192.00 & 26.06 & 87.53 & 4156 \\
\hline 2 & $\begin{array}{l}\text { IR 78875-207-B- } \\
\text { B-B }\end{array}$ & 100.67 & 129.00 & 101.10 & 208.83 & 24.46 & 88.29 & 4073 \\
\hline 3 & $\begin{array}{l}\text { IR 70215-70- } \\
\text { CPA-4-1-1-3 }\end{array}$ & 109.82 & 138.16 & 106.13 & 185.00 & 27.30 & 87.48 & 4692 \\
\hline 4 & IR08N136 & 106.00 & 134.33 & 104.10 & 181.83 & 25.80 & 89.82 & 4625 \\
\hline 5 & KARAJAT-3 & 105.00 & 133.50 & 99.33 & 203.83 & 25.30 & 82.28 & 4897 \\
\hline 6 & $\begin{array}{l}\text { IR } 87377-B-B- \\
93-3\end{array}$ & 101.67 & 129.67 & 107.13 & 215.67 & 23.67 & 87.65 & 4363 \\
\hline 7 & $\begin{array}{l}\text { IR 79246-105-2- } \\
2-4\end{array}$ & 108.50 & 136.67 & 107.43 & 179.17 & 26.06 & 83.94 & 4164 \\
\hline 8 & BRRI Dhan-38 & 91.83 & 120.33 & 99.53 & 204.33 & 21.80 & 88.00 & 3286 \\
\hline 9 & Swarna Sub-1 & 120.00 & 147.33 & 93.50 & 144.50 & 22.30 & 80.22 & 4366 \\
\hline 10 & Sabitri & 112.50 & 139.83 & 103.50 & 211.00 & 24.96 & 89.80 & 4515 \\
\hline 11 & Kanchhi Masuli & 109.67 & 137.67 & 113.13 & 228.00 & 22.86 & 92.00 & 4705 \\
\hline 12 & $\begin{array}{l}\text { NR 1190-24-4- } \\
2-2-2-3-2\end{array}$ & 117.00 & 144.50 & 139.60 & 174.50 & 25.03 & 88.77 & 4861 \\
\hline \multirow[t]{7}{*}{13} & Ceiharang Sub 1 & 102.67 & 131.00 & 94.90 & 180.33 & 22.03 & 91.75 & 4133 \\
\hline & Grand Mean & 106.89 & 134.98 & 105.73 & 196.07 & 24.43 & 87.50 & 4372 \\
\hline & F test Genotype & $* *$ & $* *$ & $* *$ & NS & $* *$ & $* *$ & $* *$ \\
\hline & Year & NS & $* *$ & $* *$ & NS & $* *$ & NS & $* *$ \\
\hline & Genotype x Year & NS & $*$ & NS & NS & NS & NS & NS \\
\hline & $\mathrm{CV} \%$ & 2.14 & 1.71 & 4.91 & 17.39 & 5.58 & 5.24 & 8.62 \\
\hline & LSD (0.05) & 3.531 & 3.561 & 8.03 & & 2.108 & 7.09 & 582.4 \\
\hline
\end{tabular}

Note: $* *=\mathrm{p}<0.01, *=\mathrm{p}<0.05$

\section{Conclusion}

In CVT of 2014-15, NR 2158-13-1-2-4-1, Cieharang Sub-1 and NR 2157-166-2-1-1-1identified as promising genotypes by producing 25 and 4 percent higher grain yield than grain yield of national average and check variety, Sabitri respectively. Regarding maturity days, Cieharnag Sub-1 was found early (130 days) than NR 2158-13-1-2-4-1 (139 days) and NR 2157-166-2-1-1-1 (143 days). Similarly, highest grain filling was observed in Cieharang Sub-1 (90.37\%) followed by NR 2157-166-2-1-1-1 (87.77\%) and NR 2158-13-1-2-4-1(86.37\%). These three genotypes are identified as promising and are recommended to include in regional varietal trial from coming rice season to develop as regional varieties.

In RVT of 2014-15, KARAJAT-3 and NR 1190-24-4-2-22-3-2 genotypes produced 32 and 8 percent higher grain yield than the yield of national average and check variety, Sabitri respectively. In case of days to maturity, KARAJAT-3 was found a week early and NR 1190-24-4-22-2-3-2 5 about a week late than Sabitri. Grain filling of these high yielding genotypes was found 82 and 88 percent respectively. Panicle length of these genotypes were found $25 \mathrm{~cm}$. So, these two genotypes identified as promising and are recommend to include in farmer's field trial for further verification of grain yield in farmers' managed condition as well to different agro-environment of eastern Terai.

\section{Acknowledgement}

Authors are grateful to the Regional Director Dr BN Chaudhary and present Regional Director Dr. HK Shrestha for their administrative support in conducting experiments. The authors express heartfelt thanks to the field staffs of RARS, Tarahara for their untiring efforts in conducting experiments in the field. National Rice Research Program, Hardinath, Dhanusha also deserve special thanks for supporting genetic material.

\section{References}

ADS (2014) Agriculture Development Strategy, Ministry of Agriculture and Development, Singh Durbar, Kathmandu.

Editorial Nature genetics (2009) The cup half empty. Nature genetics 41: 6. DOI: 10.1038/ng0609-635

Ghimire S, Dhungana S, Krishna W, No T and Sherchan DP (2013) Biophysical and Socio-economic Characterization of Cereals Production Systems of Central Nepal. SocioEconomic Program Working Paper No 9, Mexico, CIMMYT

IRRI (2006) Bringing hope, improving lives: Strategic plan, $2007-$ 2015, Manila. 61p. 
Juliano BO (1994) Rice Chemistry and Technology. The American Association of Cereal Chemists, Inc. St. Paul, Minnesta, USA, $2^{\text {nd }}$ Printing.

Mallik RN (1988) Rice in Nepal. Kala Prakashan, Nepal

MOAD (2014) Statistical information on Nepalese agriculture. Ministry of Agriculture Development. Agri-Business Promotion and Statistics Division, Singh Durbar, Kathmandu.

OECD (1999) Consensus document on biology of Oryza sativa. Report No. ENV/JM/MONO 99(26), OECD. Environment health and safety publication, Paris.

Oka HI (1988) Origin of cultivated rice. Elsevier, Amsterdam
RARS (2014) Annual Report. Regional Agricultural Research Station, Tarahara, Sunsari, Nepal, NARC Publication Serial Number: 00153-63/2014/2015.

Takahashi N (1984) Seed germination and seeding growth. In: Tsonoda S and Takahashi N (eds.) Biology of rice, Vol 7, Elsevier, Amsterdam. DOI: 10.1016/B978-0-444-996152.50008-9

Yadav RB, Mahato RK, Akhtar T, Mishra KK, Yadav DR,, Yadav S, Sharma P and Sah S (2013). Varietal Development for Rice under Irrigated Ecosystem during 2009-10. In: Proceedings of the 27th National Summer Crops Workshop (Vol-1). Organized by Nepal Agricultural Research Council, Singh durbar Plaza, Kathmandu 